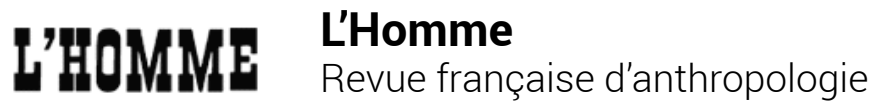

183 | 2007

Comment être parents ?

Donna A. Buchanan, Performing Democracy:

Bulgarian Music and Musicians in Transition

Chicago, University of Chicago Press, 2006

\section{Olivier Féraud}

\section{(2) OpenEdition \\ Journals}

Édition électronique

URL : http://journals.openedition.org/lhomme/9751

DOI : 10.4000/lhomme.9751

ISSN : 1953-8103

Éditeur

Éditions de l'EHESS

Édition imprimée

Date de publication : 1 septembre 2007

Pagination : 221-224

ISSN : 0439-4216

Référence électronique

Olivier Féraud, « Donna A. Buchanan, Performing Democracy : Bulgarian Music and Musicians in Transition », L'Homme [En ligne], 183 | 2007, mis en ligne le 28 juin 2007, consulté le 24 septembre 2020. URL : http://journals.openedition.org//homme/9751 ; DOI : https://doi.org/10.4000//homme. 9751

Ce document a été généré automatiquement le 24 septembre 2020.

(c) École des hautes études en sciences sociales 


\title{
Donna A. Buchanan, Performing Democracy: Bulgarian Music and Musicians in Transition
}

\author{
Chicago, University of Chicago Press, 2006
}

\section{Olivier Féraud}

1 L'OUvRAge de Donna A. Buchanan est un parcours politique et historique qui, à travers les pratiques musicales "néotraditionnelles", trace un axe de lecture privilégié des processus de construction identitaire et de réinvention de la tradition engagés par les bouleversements qu'a connus la Bulgarie dans les dernières décennies du $\mathrm{xx}^{\mathrm{e}}$ siècle. L'ensemble du livre rend compte d'une longue enquête ethnographique menée entre 1986 et 1996, et dessine l'analyse sociopolitique de cette période clé que l'auteur nomme «transition ».

2 L'originalité de Performing Democracy ne réside pas dans le caractère inédit de l'objet d'étude, puisque, au moment où Donna A. Buchanan terminait son enquête, paraissait l'ouvrage de Timothy Rice, May it Fill your Soul ${ }^{1}$, qui constituait déjà un large panorama des pratiques musicales bulgares et des processus de transmission. La particularité de l'ouvrage de Donna A. Buchanan est avant tout son inscription dans un champ politique fort, qui est celui des vastes changements politiques survenus lors des événements de 1989, c'est-à-dire le passage du socialisme au postsocialisme après la chute du mur de Berlin et la construction d'un système démocratique. Une seconde particularité est l'étendue du champ d'investigation: c'est au travers de ce que l'ethnomusicologie française a coutume d'appeler le processus de folklorisation que l'auteur traite de bien des aspects de la société bulgare. Qu'une recherche soit conduite sur ces deux tableaux est une qualité qu'il convient de saluer : une ethnographie minutieuse menée auprès de musiciens et une vaste mise en problématique dans le contexte politique, social et économique. C'est en effet dans un constant face à face que ces deux dimensions se répondent : pratiques musicales et valorisations politiques se dévoilent et se meuvent dans cette même «transition ». 
3 L'ouvrage est organisé en trois grandes parties. La première, "Socialist Culture in Transition ", expose le contexte ethnographique et politique ainsi que les orientations théoriques de la recherche. Elle expose d'emblée le propos ethnomusicologique en expliquant comment des musiciens professionnels, formés au «neotraditional socialist musical canon », ont remplacé des pratiques musicales qui étaient traditionnellement à la charge des musiciens amateurs issus du monde rural. Présentés comme "the most visible and nationalist symbol of the socialist culture", ces "folk song and dance ensembles ", pour la plupart financés par l'État, constituent pour l'auteur une entrée privilégiée vers la compréhension des interactions complexes qui se sont jouées dans la société bulgare durant tout le $\mathrm{xx}^{\mathrm{e}}$ siècle : entre la musique (ses pratiques, ses acteurs et ses mises en scène), les valeurs et les mesures prises par les pouvoirs politiques, et enfin les enjeux identitaires. Créés dès les années 1950 sur les bases d'un modèle soviétique, dont la politique reposait sur l'inscription dans une construction culturelle balkanique puisant dans des racines toujours plus locales et "authentiques", ces ensembles prennent la forme fixe d'un orchestre à l'allure symphonique : un chœur composé de femmes, un orchestre masculin d'instruments inspirés de l'organologie traditionnelle et un ensemble mixte de danseurs qui interprètent sur la scène ce qui n'est autre qu'une stylisation des chorégraphies villageoises. Jusqu'aux événements de 1989, ces ensembles étaient envoyés dans chaque ville et village comme emblème d'une identité nationale prise en charge par l'État. Ils remodelaient la manière dont musiques et danses traditionnelles étaient jusque-là pratiquées, et les réinventaient en une forme néo-traditionnelle dont la mise en spectacle reposait en même temps sur une mise en scène de deux idéologies : les images d'une authenticité pastorale, d'une part, et les valeurs élitistes du classicisme ouest-européen, de l'autre. Dans cette partie, l'auteur décrit l'évolution du statut de musicien et l'histoire des orchestres professionnels, analyse le système de l'administration culturelle et la manière dont elle supervisait les orchestres.

4 Après cette longue introduction, on entre de plain-pied dans l'enquête ethnographique, au moyen d'un style narratif qui prend parfois la forme d'un journal de bord quasi autobiographique, mêlant récits anecdotiques et analyse du contexte économique de la scène musicale officielle. En effet, la deuxième partie, "Nationalist Narratives: Marketing Bulgarian Identities through Folk Ensemble Tableaus », met au clair comment les choix esthétiques de la mise en scène ainsi que le répertoire révèlent les représentations fluctuantes des notions de tradition et d'authenticité, et surtout dessinent l'idée d'une nation bulgare. Le lecteur suit une communauté de musiciens employés par le National Ensemble for Folk Songs and Dances-Phillip Koutev et par le Ensemble for Folk Songs of the Bulgarian Radio, deux grandes compagnies nationales de «chants et danses folkloriques ». L'auteur montre comment à la fois les musiciens, les chefs d'ensemble et les commissions officielles avec lesquelles ils ont dû négocier, ont su utiliser une culture musicale d'avant guerre issue des pratiques villageoises pour créer un style nouveau qui permet en lui-même de lire à la fois les changements radicaux et les choix politiques, sociaux et économiques des deux dates charnières de l'histoire de la Bulgarie : la sujétion dès 1944 au totalitarisme socialiste soviétique et la transition brutale en une démocratie parlementaire après la chute du mur de Berlin. Si l'intention d'une "troupe nationale » et officielle comme le Koutev Ensemble est bien de préserver «the purity of the folklore style » (p. 231) et de promouvoir l'originalité et la singularité des traditions bulgares, il s'agit de passer de la place du village à la scène internationale, car chaque troupe doit savoir développer sa propre ligne artistique et 
ainsi être novatrice. Or, il apparait que de telles tensions entre le local et le national, comme l'utilisation d'une caractéristique régionale en tant qu'argument de la singularité artistique de l'ensemble, ont constitué parallèlement une résistance à la doctrine d'une nation-État monoethnique.

Donna A. Buchanan met donc en scène les stratégies que ces musiciens ont su développer en s'adaptant à un contexte mouvant, et dévoile comment, en ajustant le style et le genre de l'interprétation, ils ont su remodeler, imaginer, réinventer, encoder et rejouer les éléments du passé et ses attributs idéologiques (représentation de l'histoire, des traditions et de l'authenticité), et ont su négocier avec les philosophies politiques de la démocratie, du socialisme et du communisme soviétique. Elle nous montre ainsi en quoi, en Bulgarie plus que dans les autres États post-soviétiques, ces ensembles de musiciens professionnels ont servi d'icônes particulièrement ostentatoires d'une mise en scène de ces transitions. Comme le mentionne la légende d'une photographie parue le 8 juillet 1988 dans le Rabotnichesko Delo (journal du BKP, le parti communiste bulgare), représentant deux jeunes femmes revêtues de costumes traditionnels, "Folklore is not only an epistle from the past, but is also source of inspiration». Relié avec le message de propagande du parti inscrit plus haut " restructuring of the cultural sphere", cela suggère clairement que, selon l'auteur, "tradition would inspire the nation in its transition to a more democratic society" (p. 14).

6 La troisième grande partie, "Ethnography and Antistructure", enquête plus spécifiquement sur la récente commercialisation de la culture musicale bulgare, symptomatique de la transition vers les idéaux démocratiques du pluralisme politique et du libéralisme. Le passage du système des grands ensembles financés par l'État au circuit international, plus libéral et moins supervisé («trafficked»), a impliqué un mode de vie bien différent pour ces musiciens par rapport à ce qu'ils avaient connu jusque-là. L'ethnographie se tourne alors vers d'autres troupes dont certaines sont devenues célèbres à l'échelle internationale comme "The Bulgarian State Vocal Choir "Le mystère des voix bulgares" ", dont le bilinguisme de l'appellation marque en luimême une nette volonté d'appartenance européenne. L'éclatement des diversités musicales et culturelles qu'a connu cette période récente est marqué, entre autres, par l'apparition d'une scène de "pop song" d'influences turque et rom, et d'un genre populaire nouveau de chanson néotraditionnelle "authored Macedonian folklore». L'apparition d'éléments comme la notion d'auteur, le mouvement « ethnopop » mettant l'accent sur l'ancrage dans des racines plurielles, est alors présentée comme une affirmation des «identités sociomusicales » et de la recherche d'autres affiliations culturelles, à la fois balkaniques et turques. Pour Donna A. Buchanan, ces nouvelles affiliations marquent la rupture sociopolitique de cette "période démocratique » en ce qu'elles ne sont plus élaborées par une politique d'État mais construites par des choix adoptés par les acteurs de la scène musicale eux-mêmes.

7 Un point fort du travail de Donna A. Buchanan est qu'elle parvient à échapper à un stéréotype encore parfois présent dans l'ethnomusicologie actuelle, qui consiste à créer ou accentuer une dichotomie entre des pratiques considérées comme authentiques provenant de petites communautés rurales (position historique d'une ethnographie à la vision " pastoraliste » des traditions) et celles générées par une modernisation qui en serait une altération urbaine. D'autres dichotomies pouvant sembler elles aussi stéréotypées sont mises en lumière, comme celles de ouest/est, classique/traditionnel 
(" classical vs Folk»), ou socialiste et communiste/démocratique. L'auteur nous explique que si ces oppositions ont pu être manipulées par les idéologies politiques, les musiciens eux-mêmes en ont été les acteurs au sens où ces "folk ensembles" établissent des passerelles dans une société qui a construit elle-même ses paradoxes. La forme et le contenu de ces pratiques musicales portent en eux des éléments de ces oppositions, et considérer ces musiciens comme des agents de la transition participant à créer des formes innovantes et hybrides prend alors tout son sens dans ce contexte.

8 La présence d'un cédérom encarté dans la couverture est une qualité qui, même si cela devrait être systématique dans les éditions ethnomusicologiques, n'est encore que trop rarement prise en compte dans les choix éditoriaux. La partie interactive du cédérom comporte plusieurs sections contenant les figures citées dans le texte. Une galerie de 75 photographies présente des portraits de personnages clés, des ensembles musicaux et des instruments, et propose une visite de l'architecture politique ou historique de plusieurs villes. Une deuxième section d'illustrations présente une carte des régions ethnographiques de la Bulgarie, des organigrammes rendant compte des structures administratives et des schémas représentant la composition des orchestres, l'ambitus ou le doigté de quelques instruments de musique. Enfin, la troisième section thématique est consacrée à des transcriptions musicales et textuelles, dont certaines ont été réalisées et traduites par l'auteur. Plusieurs d'entre elles sont les transcriptions des exemples musicaux qui peuvent être écoutés, et l'on peut noter l'aspect pédagogique de la juxtaposition sur la même page d'une interface permettant d'écouter l'exemple tout en suivant la transcription. On ne peut que se réjouir de cet ajout sonore, même si l'on déplore le caractère peut-être trop didactique de la sélection des extraits musicaux, au sens où elle ne semble constituer qu'un panorama historique illustrant le propos. L'écoute des exemples musicaux n'apparait que secondaire devant une écriture imposante, comme l'illustre la brièveté des séquences dont beaucoup sont shuntées au début et à la fin.

9 En conclusion, la parution de Performing Democracy ne peut être que bienvenue dans une période où l'ethnomusicologie, à l'image d'autres disciplines de plus en plus considérées comme mineures, a besoin de tels ouvrages aidant à la désenclaver et à l'inscrire dans des problématiques géopolitiques plus vastes. On en profitera en effet pour rappeler que, même si l'étude des processus de folklorisation est plus que tout autre un terrain fertile pour repenser la discipline, il est aujourd'hui nécessaire que dans son ensemble l'ethnomusicologie s'inscrive dans les problématiques de changement et de réactualisation des pratiques musicales. Une oeuvre synthétique comme celle de Donna A. Buchanan constitue donc à présent un élément important du paysage littéraire ethnomusicologique. Même si son ethnographie reste limitée à la scène musicale bulgare, le propos s'étend aux problématiques plus vastes des dynamiques musicales balkaniques. Un tel ouvrage ne peut que poser des bases méthodologiques, théoriques et épistémologiques pour la compréhension toujours plus actuelle des processus de folklorisation, de mise en scène ou d'instrumentalisation des identités, de création et de réinvention des cultures musicales. 


\section{NOTES}

1. Timothy Rice, May it Fill your Soul : Experiencing Bulgarian Music, Chicago, University of Chicago Press, 1994.

\section{AUTEUR}

\section{OLIVIER FÉRAUD}

EHESS, Laboratoire d'anthropologie et d'histoire de l'institution de la culture, Paris. olivierferaud@free.fr 